\title{
Esterco bovino e biofertilizante no cultivo de erva-cidreira-verdadeira (Melissa officinalis L.)
}

\begin{abstract}
SANTOS, M.F. ${ }^{1}$; MENDONÇA, M.C. ${ }^{2 \star}$; CARVALHO FILHO, J.L.S. ${ }^{3}$; DANTAS, I.B. ${ }^{3}$; SILVA-MANN, R. ${ }^{4}$; BLANK, A.F. ${ }^{4}$ ${ }^{1}$ Escola Superior de Agricultura "Luiz de Queiroz" - ESALQ/USP, Departamento de Genética, Av. Pádua Dias, 11Caixa Postal 83, CEP: 13400-970, Piracicaba-Brasil. ${ }^{2}$ Emdagro/Embrapa Tabuleiros Costeiros, Av. Beira Mar, 3250, 49025-040, Aracaju-Brasil. * marcelom@cpatc.embrapa.br. ${ }^{3}$ Universidade Federal de Lavras - UFLA, Departamento de Agricultura, Caixa Postal 37, CEP: 37200-000, Lavras-Brasil. ${ }^{4}$ Departamento de Engenharia Agronômica - UFS; Av. Marechal Rondon s/n, J. Rosa Elze, CEP: 49100-000, São Cristóvão-Brasil.
\end{abstract}

\begin{abstract}
RESUMO: A importância fitoterápica e, consequentemente, econômica da Melissa officinalis tem contribuído para expansão do cultivo. O óleo essencial das folhas é formado por constituintes químicos que podem ser largamente empregados na indústria farmacêutica por possuírem atividades antioxidativa, antivirótica e sedativa. Assim sendo, objetivou-se no presente trabalho avaliar o efeito de combinações de doses de adubação orgânica com biofertilizante comercial e esterco bovino no cultivo de Melissa officinalis. Os tratamentos foram constituídos de quatro doses de biofertilizante orgânico (Vitassolo ${ }^{\circledR}$ ) e esterco bovino, sendo estes de 0, 30.000, 60.000 e $90.000 \mathrm{~L} \mathrm{ha}^{-1}$. Os tratamentos foram arranjados no delineamento experimental blocos casualizados no fatorial $4 \times 4$, com 3 repetições. As características avaliadas foram altura de planta, rendimento de folha, teor e rendimento de óleo essencial. As maiores doses de esterco bovino resultaram em um maior desenvolvimento das plantas, em relação às mesmas doses do biofertilizante.
\end{abstract}

Palavras-chave: combinação de fertilizantes, plantas medicinais, óleo essencial

\begin{abstract}
Cattle manure and biofertilizer on the cultivation of lemon balm (Melissa officinalis L.). The phytotherapic and economic importance of Melissa officinalis has contributed to its cultivation expansion. The essential oil of leaves is composed of chemical constituents, which can be largely employed in the pharmaceutical industry due to their antioxidant, antivirotic and sedative activities. Thus, the aim of the present work was to evaluate the effect of level combination of organic fertilization with commercial biofertilizer and cattle manure on Melissa officinalis cultivation. Treatments constituted of four levels of organic biofertilizer (Vitassolo ${ }^{\circledR}$ ) and cattle manure at $0 ; 30,000 ; 60,000$ and $90,000 \mathrm{~L} \mathrm{ha}^{-1}$. Experimental design was in randomized blocks, in split plot $4 \mathrm{X} 4$ factorial arrangement, with three replicates. The characteristics evaluated were plant height, leaf yield, essential oil content and yield. The highest cattle manure levels resulted in higher plant development, compared with the same biofertilizer levels.
\end{abstract}

Key words: fertilizer combination, medicinal plants, essential oil

\section{INTRODUÇÃO}

A importância fitoterápicae, consequentemente, econômica da Melissa officinalis tem contribuído para expansão do seu cultivo. O óleo essencial das folhas é formado por constituintes químicos amplamente empregados na indústria farmacêutica por possuírem atividades antioxidativa, antibiótica, antifúngica, antibacteriana e sedativa (Tekel et al., 1997).

As espécies medicinais e aromáticas apresentam melhor desenvolvimento onde os solos possuem características favoráveis para a expansão do sistema radicular (Chaves, 2002). Ressalta-se que uma das práticas culturais para melhorar as qualidades físico-químicas e microbiológicas do solo é a utilização da adução orgânica (Kiehl, 1985).

Os resíduos orgânicos liberam os nutrientes para as plantas mais lentamente que os adubos

Recebido para publicação em 08/11/2006

Aceito para publicação em 31/07/2009

Rev. Bras. PI. Med., Botucatu, v.11, n.4, p.355-359, 2009. 
químicos, porém essa liberação é realizada constantemente resultando em benefícios não só químicos, mas também físicos para o solo, como a melhoria na estruturação, aeração e drenagem, e retenção de água, além de outros efeitos sobre as propriedades físico-químicas do solo (Kiehl, 1985; Ming, 1994; Rajeswara Rao, 2001).

O uso de adubação orgânica no cultivo de mil folhas (Achillea millefolium L.) proporcionou maior incremento de biomassa e rendimento de óleo essencial (Scheffer, 1998). Cruz (1999), estudando hortelã-rasteira (Mentha vilosa Huds.), verificou que na estação seca a dose de $2,0 \mathrm{~kg} \mathrm{~m}^{-2}$ de esterco bovino melhorou o rendimento de óleo essencial, mas não apresentou influência sobre a massa seca.

Os aspectos abordados na literatura sobre a Melissa officinalis L., em grande parte, tratam da composição química e qualidade do óleo essencial, propriedades farmacêuticas da planta, dentre outros. Todavia, são poucas as informações disponíveis sobre seus aspectos agronômicos, tais como época adequada para plantio e colheita, adubação, espaçamento, podas e controle fitossanitário (Scheffer, 1992). Há necessidade de estudos sobre as espécies medicinais quando submetidas às técnicas de produção sem influenciar o valor terapêutico da planta, isso, considerando-se o fato de que os princípios ativos podem sofrer alterações conforme as técnicas de cultivo (Madueño Box, 1973).

Este trabalho teve como objetivo avaliar 0 rendimento de folhas, altura de plantas e o teor e rendimento de óleo essencial de erva-cidreiraverdadeira em resposta a doses e combinação de adubos orgânicos.

\section{MATERIAL E MÉTODO}

O experimento foi realizado no Campus Rural da Universidade Federal de Sergipe/DEA, localizado no município de São Cristóvão - SE, cujo solo é classificado como Argissolo Vermelho Amarelo distrófico, textura média. A análise química do solo da área experimental apresentou as características descritas na Tabela 1.

Os tratamentos foram constituídos de quatro doses de biofertilizante orgânico (Vitassolo ${ }^{\circledR}$ ) e quatro de esterco bovino: 0, 30.000, 60.000 e $90.000 \mathrm{~L} \mathrm{ha}^{-1}$. Os tratamentos foram arranjados no delineamento experimental blocos casualizados no fatorial $4 \times 4$, com três repetições.

Sementes comerciais de melissa (ISLA) foram semeadas em bandejas de PVC com substrato de húmus de minhoca e terra vegetal. Trinta dias após a semeadura, as plântulas foram transplantadas para tubetes $\left(110 \mathrm{~cm}^{3}\right)$ contendo mistura de substrato $1: 1$ de esterco bovino e húmus de minhoca, mais $40 \%$ de pó de coco.
TABELA 1. Resultados das análises químicas do Argissolo Vermelho Amarelo distrófico textura média, do município de São Cristóvão, SE.

\begin{tabular}{|c|c|}
\hline Características & Valores \\
\hline $\mathrm{pH}\left(\mathrm{CaCl}_{2}\right)$ & 5,5 \\
\hline $\mathrm{MO}\left(\mathrm{dag} \mathrm{\textrm {m } ^ { - 3 } )}\right.$ & 1,5 \\
\hline $\mathrm{P}$ disponível $\left(\mathrm{mg} \mathrm{cm}^{3}\right)$ & 49,0 \\
\hline $\mathrm{K}\left(\mathrm{meq} \mathrm{dm}^{-3}\right)$ & 0,22 \\
\hline $\mathrm{Ca}\left(\right.$ meq $\left.\mathrm{dm}^{-3}\right)$ & 2,6 \\
\hline $\mathrm{Mg}\left(\right.$ meq dm $\left.{ }^{-3}\right)$ & 0,9 \\
\hline Al trocável (meq dm $\left.{ }^{-3}\right)$ & 1,7 \\
\hline $\mathrm{SB}\left(\right.$ meq $\left.\mathrm{dm}^{-3}\right)$ & 3,7 \\
\hline$V(\%)$ & 47 \\
\hline CTC (meq $\left.\mathrm{dm}^{-3}\right)$ & 5,5 \\
\hline
\end{tabular}

Após aração e gradagem, fez-se calagem (calcário dolomítico $\mathrm{Ca}: \mathrm{Mg}=4: 7$ ) para elevar a saturação de base a $70 \%, 20$ dias antes da adubação orgânica com esterco bovino e biofertilizante orgânico (Vitassolo ${ }^{\circledR}$ ). Na Tabela 2 constam os resultados da análise química para os dois fertilizantes.

Cada parcela tinha $1,80 \mathrm{~m}^{2}(1,80 \mathrm{~m} \times 1,0$ $\mathrm{m})$, com 24 plantas, sendo oito plantas úteis por parcela. O transplante das mudas ao campo foi feito quando elas tinham $13 \mathrm{~cm}$ de altura no espaçamento de $30 \mathrm{~cm} \times 25 \mathrm{~cm}$. A irrigação foi pelo sistema de gotejamento.

As características foram avaliadas uma única vez aos 120 dias após o plantio, correspondendo ao final do ciclo da cultura. Por ocasião da colheita da parte aérea das plantas, realizada no período da manhã, avaliou-se a altura de planta, por meio da amostragem aleatória de três plantas da parcela útil que foram medidas desde sua base até a última folha. Após a colheita, as plantas foram transferidas para o galpão de beneficiamento visando sua separação de impurezas e misturas com espécies indesejadas. A parte aérea de todas as plantas das parcelas foi cortada no período da manhã e transferida para estufa à temperatura de $40^{\circ} \mathrm{C}$ por um período de cinco dias. Para avaliação do rendimento, os ramos e as folhas secas foram separados e pesados. Uma amostra de $100 \mathrm{~g}$ das folhas secas foi separada para a extração do óleo essencial por meio da hidrodestilação, visando obter os dados de teor ( $\mathrm{mL} 100 \mathrm{~g}^{-1}$ de folha) e rendimento de óleo (t ha-1).

Os resultados foram submetidos à análise de variância e as médias comparadas pelo teste de Tukey $(p \leq 0,05)$, por meio do Programa Estatístico SAEG v. 5.0 UFV - MG. Os resultados referentes aos efeitos dos níveis de adubação, das características 
TABELA 2. Resultado da análise química do biofertilizante comercial Vitassolo ${ }^{\circledR}$ e esterco bovino.

\begin{tabular}{|c|c|c|}
\hline Características & Vitassolo $^{\circledR}$ & Esterco bovino \\
\hline$\overline{\mathrm{pH}}$ (em água) & $\bar{x}$ & 8,3 \\
\hline$P$ & $x$ & 20,6 $\left(\right.$ mehlich $\left.^{-1}\right)\left(\mathrm{mg} \mathrm{dm}^{-3}\right)$ \\
\hline P-remanescente & $x$ & $50,4\left(\mathrm{mg} \mathrm{L}^{-1}\right)$ \\
\hline $\mathrm{K}^{+}$ & 15,38 (meq/100g t.f.s.a.) & $100\left(\mathrm{mg} \mathrm{dm}^{-3}\right)$ \\
\hline $\mathrm{Na}^{+}$ & 6,69 (meq/100g t.f.s.a.) & $3,0\left(\mathrm{cmol}_{\mathrm{c}} \mathrm{dm}^{-3}\right)$ \\
\hline $\mathrm{Ca}^{++}$ & - & $3,0\left(\mathrm{cmol}_{\mathrm{c}} \mathrm{dm}^{-3}\right)$ \\
\hline $\mathrm{Mg}^{++}$ & - & $8,9\left(\mathrm{cmol}_{\mathrm{c}} \mathrm{dm}^{-3}\right)$ \\
\hline $\mathrm{Al}^{+++}$ & $x$ & $0,0\left(\mathrm{cmol}_{\mathrm{C}} \mathrm{dm}^{-3}\right)$ \\
\hline $\mathrm{H}+\mathrm{Al}$ & 0,78 (meq/100g t.f.s.a.) & $0,7\left(\mathrm{cmol}_{\mathrm{c}} \mathrm{dm}^{-3}\right)$ \\
\hline SB & - & $15,2\left(\mathrm{cmol}_{\mathrm{c}} \mathrm{dm}^{-3}\right)$ \\
\hline $\mathrm{t}$ & - & $15,2\left(\mathrm{cmol}_{\mathrm{c}} \mathrm{dm}^{-3}\right)$ \\
\hline $\mathrm{T}$ & 34,10 (meq/100g t.f.s.a.) & $15,9\left(\mathrm{cmol}_{\mathrm{C}} \mathrm{dm}^{-3}\right)$ \\
\hline V & $97,71 \%$ & $95,6(\%)$ \\
\hline $\mathrm{m}$ & - & $0(\%)$ \\
\hline MO & - & $15,5\left(\mathrm{dag} \mathrm{kg}^{-1}\right)$ \\
\hline $\mathrm{Zn}$ & 2,78 ppm & $19,2\left(\mathrm{mg} \mathrm{dm}^{-3}\right)$ \\
\hline $\mathrm{Fe}$ & $26,55 \mathrm{ppm}$ & $500,0\left(\mathrm{mg} \mathrm{dm}^{-3}\right)$ \\
\hline $\mathrm{Mn}$ & 50,43 ppm & $165,0\left(\mathrm{mg} \mathrm{dm}^{-3}\right)$ \\
\hline $\mathrm{Cu}$ & 2,86 ppm & $0,5\left(\mathrm{mg} \mathrm{dm}^{-3}\right)$ \\
\hline B & - & $12,5\left(\mathrm{mg} \mathrm{dm}^{-3}\right)$ \\
\hline$S$ & - & $386,9\left(\mathrm{mg} \mathrm{dm}^{-3}\right)$ \\
\hline
\end{tabular}

avaliadas, foram submetidos à análise de regressão no Programa Table Curve 2D/3D v.

\section{RESULTADO E DISCUSSÃO}

De uma maneira geral, as plantas apresentaram bom desenvolvimento e produtividade em reposta à adubação orgânica. Embora tenha sido verificado efeito significativo na interação biofertilizante (Vitassolo) e esterco bovino para rendimento de folha e altura de planta (Figuras 1A e 1B) o efeito individual do esterco bovino foi superior para o rendimento de óleo.

Maiores concentrações de esterco bovino e VitassoloÓ proporcionaram um efeito crescente para altura de planta (Figura 1).

Sousa et al. (2003) avaliando a produtividade de erva cidreira com diferentes tipos de adubação (esterco bovino, húmus, composto orgânico e NPK), em casa de vegetação, obtiveram resultados semelhantes, ou seja, o esterco bovino apresentou melhores resultados no que diz respeito à produtividade da parte aérea da planta de erva cidreira.

Chaves (2002) verificou em alfavaca cravo
(Ocimum gratissimum L.), que doses crescentes de adubo orgânico associadas à maior idade na época de colheita da planta favoreceu o aumento de produção de folhas, caules e inflorescências, alcançando a maior produtividade com a dose de $12 \mathrm{~kg} \mathrm{~m}^{-2}$ de adubo orgânico.

Maia et al. (2004) avaliaram a influência da adubação orgânica e mineral no crescimento de Hyptis suaveolens (L.) Poit., constatando que os maiores acúmulos de biomassa seca de folha foram obtidos com a aplicação de esterco de aves (45 e $80 \mathrm{~g}$ ), e os menores foram da testemunha $(3 \mathrm{e} 13 \mathrm{~g})$. Para hortelã japonesa (Mentha arvensis L. var. piperancens Moor), o uso de $6 \mathrm{~kg} \mathrm{~m}^{-2}$ de esterco bovino resultou na maior produção de massa seca de parte aérea $(4,74 \mathrm{t}$ ha-1 $)$ das plantas, em relação à testemunha (3,55 t ha-1); no entanto, o uso de $8 \mathrm{~kg} \mathrm{~m}^{-2}$ resultou em produção

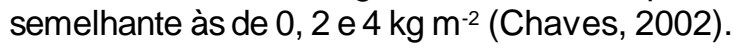

Rajeswara Rao (2001) estudou a influência de diferentes níveis de esterco de curral $\left(0 \mathrm{e} 15 \mathrm{t}_{\text {ano-1 }}\right) \mathrm{e}$ fertilizante nitrogenado $\left(0,40,80 \mathrm{Kg}^{-1} \mathrm{ha}^{-1} \mathrm{~N}\right.$ por ano) na biomassa e rendimento do óleo essencial de palmarosa (Cymbopogon martinii, Roxb. wats. var. 
(A)

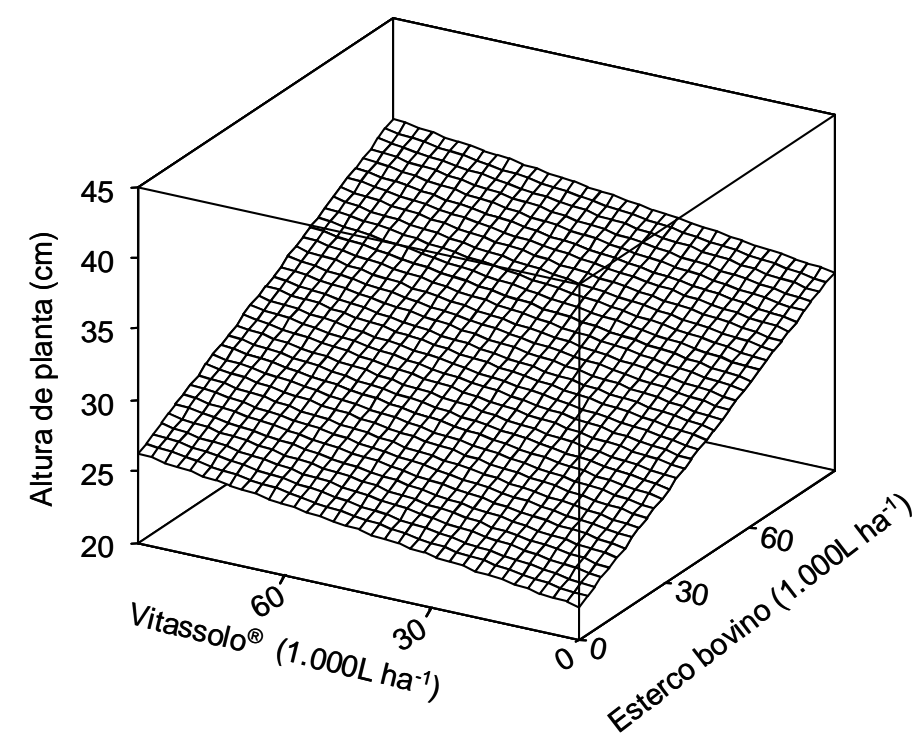

$y=22,3055+0,1296^{* *} e b+, 044^{*}$ vs $\quad R^{2}=0,82$

(B)

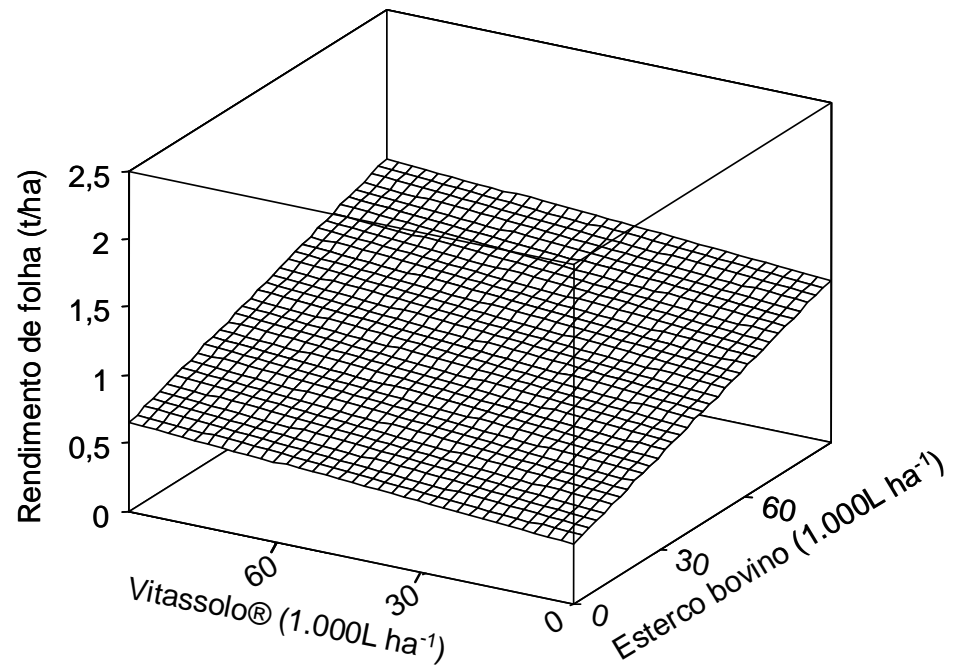

$y=0,4445+0,0083^{* *} e b+0,00234^{b}$ vs $\quad R^{2}=0,54$

(C)

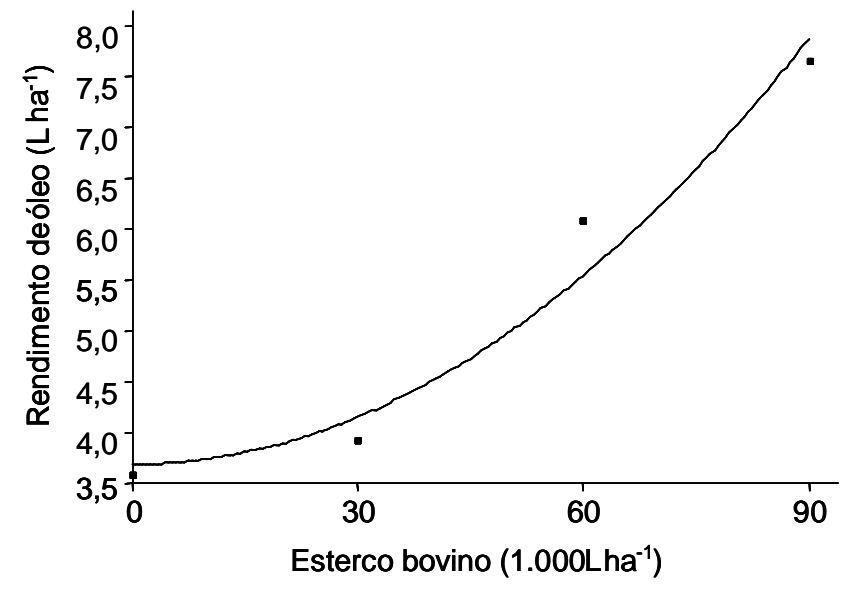

$y=3,679+0,00051^{\star *} e b^{2} \quad R^{2}=0,96$

FIGURA 1. Efeito de doses e combinações de adubo orgânico [biofertilizante - Vitassolo (vs) e esterco bovino (eb)] na altura de planta (A), rendimento de folha (B) e rendimento de óleo essencial (C), no cultivo de $M$. officinalis. **, ,, b Significativo a $1 \%, 5 \%$ e $25 \%$ de probabilidade pelo teste t. 
motia). Durante o período do experimento, foram realizadas sete colheitas em dois anos com rendimento de biomassa total de 23.6 - $37.2 \mathrm{t} \mathrm{ha}^{-1}$, e rendimento de óleo essencial de 99,2 - 159,1 $\mathrm{kg} \mathrm{ha}^{-1}$. A aplicação de $15 \mathrm{t} \mathrm{ha}^{-1}$ por ano de esterco bovino induziu o aumento do rendimento total de biomassa em $10,7 \%$ e o rendimento total de óleo essencial em $10,3 \%$ em relação à testemunha. Este fato pode estar relacionado à quantidade de nitrogênio presente no esterco, sendo este nutriente o responsável pelo crescimento vegetativo das plantas.

Observou-se efeito crescente do rendimento de óleo essencial em função das doses de esterco bovino, nas maiores concentrações (Figura 3). Trabalhos realizados por Oliveira Júnior et al. (2004) demonstraram que a aplicação de adubação organomineral sem calagem na produção de mudas de arnica (Lychnophora ericoides) favoreceu alto rendimento com alto teor de óleo, além de produção satisfatória de biomassa fresca de parte aérea. Em contrapartida, Ming (1992) verificou que à medida que se aumentou os níveis de adubo orgânico em Lippia alba houve aumento na produção de biomassa, no entanto, para rendimento de óleo essencial houve uma relação inversa aos resultados de produção de biomassa.

O teor de óleo essencial não foi influenciado pela interação biofertilizante e esterco bovino, nem pelas doses de biofertilizante. Este fato pode estar associado a menor concentração de macro e micronutrientes presentes nas fontes de adubação. Nutricionalmente, o resíduo orgânico serve como fonte de N, P, S e micronutrientes; biologicamente, influencia as atividades dos organismos da microfauna e microflora, e fisicamente, promove boa estruturação do solo aumentando a agregação, aeração e retenção de água, dentre outras (Stevenson, 1982). Shalaby \& Khattab (1993) demonstraram que doses elevadas da combinação NPK aumentaram o teor de óleo das plantas em função do aumento do diâmetro das glândulas de óleo.

\section{CONCLUSÃO}

Nas condições do presente trabalho concluise que é viável a utilização de adubação orgânica com esterco bovino e/ou vitassolo $\AA$. O esterco bovino influenciou, significativamente, o desenvolvimento e o rendimento de óleo essencial das plantas de ervacidreira-verdadeira.

\section{REFERÊNCIA}

CHAVES, F.C.M. Produção de biomassa, rendimento e composição de óleo essencial de alfavaca-cravo (Ocimum gratissimum L.) em função da adubação orgânica e épocas de corte. 2002. 144p. Tese (Doutorado) - Universidade Estadual Paulista, São Paulo.

CRUZ, G.B. Desenvolvimento de sistema de cultivo para hortelã-rasteira (Mentha $x$ vilosa Huds.). 1999. 74p. Tese (Mestrado) - Universidade Federal do Ceará, Fortaleza.

KIEHL, E.J. Fertilizantes orgânicos. Piracicaba: Agronômica Ceres, 1985. 492p.

MADUEÑO BOX, M. Cultivo de plantas medicinais. Madrid: Labor, 1973. 490p.

MAIA, S.S.S. et al. Efeito da adubação orgânica e mineral sobre o crescimento de Hyptis suaveolens (L.) Poit. Horticultura Brasileira, v.22, n.2, Supl., 2004. CD-Rom. MING, L.C. Influência da adubação orgânica na produção de biomassa, rendimento e teor de óleo essencial de Lippia alba (Mill.) N. E. Br.-Verbenaceae. 1992. 169p. Tese (Mestrado) - Universidade Federal do Paraná, Curitiba.

MING, L.C. Estudo e pesquisa de plantas medicinais na agronomia. Horticultura Brasileira, v.12, n.1, p.3-9, 1994. OLIVEIRA JÚNIOR, A.C. et al. Teor e rendimento de óleo essencial no peso fresco de arnica, em função da calagem e adubação. Horticultura Brasileira, v.23, n.3, p.735-9, 2005.

RAJESWARA RAO, B.R. Biomass and essential oil yields of rainfed palmarosa (Cymbopogon martinii (roxb.) Wats. Var. motia Burk.) supplied with different levels of organic manure and fertilizer nitrogen in semi-arid tropical climate. Industrial Crops and Products, v.14, p.171-8, 2001.

SCHEFFER, M.C. Roteiro para estudo de aspectos agronômicos das plantas medicinais selecionadas pela fitoterapia do SUS-PR/CEMEPAR. SOB Informa, v.10-11, n.1-2, p.29-31, 1992.

SCHEFFER, M.C. Influência da adubação orgânica sobre a biomassa, rendimento e composição do óleo essencial de Achillea millefolium - mil-folhas. In: MING, L.C. et al. (Coord.). Plantas medicinais aromáticas e condimentares: avanços na pesquisa agronômica. Botucatu: UNESP, 1998. v.1, p.1-22.

SHALABY, A.S.; KHATTAB, M.D. Cultivation of Melissa officinalis in Egypt. 1. Effects of fertilization, spacing and planting season. Acta Horticulturae, n.331, p.115-20, 1993. SOUSA, A.H. et al. Produção de biomassa na parte aérea da erva cidreira (Melissa ssp.) em função de doses de esterco bovino, húmus de minhoca, composto orgânico e NPK em casa de vegetação. Revista de Biologia e Ciências da Terra, v.3, n.2, 2003. Disponível em: <http:// redalyc.uaemex.mx/redalyc/pdf/500/50030207.pdf >. Acesso em: 20 jul. 2009.

STEVENSON, F.J. Humus chemistry. New York: John Wiley \& Sons, 1982. 443p.

TEKEL, J. et al. Determination of uracil herbicide residues and components of essential oil in Melissa officinalis $L$. in its main development phases. Journal of Essential Oil Research, v.9, p.63-6, 1997. 